\title{
Differential Role of Rapamycin in Epidermis-Induced IL-15-IGF-1 Secretion via Activation of Akt/mTORC2
}

\author{
Yang Baia,b Rui Xuc Xueyuan Zhang ${ }^{\mathrm{b}}$ Xiaorong Zhang ${ }^{\mathrm{a}}$ Xiaohong $\mathrm{Hu}^{\mathrm{a}}$ \\ Yashu Lia Haisheng Lia Meixi Liu ${ }^{a}$ Zhenggen Huanga Rongshuai Yan ${ }^{a}$ \\ Weifeng He $\mathrm{H}^{\mathrm{a}}$ Gaoxing Luo ${ }^{\mathrm{a}}$ Jun Wu $\mathrm{W}^{\mathrm{a}, \mathrm{d}}$
}

\begin{abstract}
aState Key Laboratory of Trauma, Burns and Combined Injury, Chongqing Key Laboratory for Proteomics Disease, Institute of Burn Research, Southwest Hospital, The Third Military Medical University, Chongqing, 'Department of Otolaryngology, Southwest Hospital, The Third Military Medical University, Chongqing, 'Department of Neurology, Xinqiao Hospital, The Third Military Medical University, Chongqing, dDepartment of Burns, the First Affiliated Hospital, Sun Yat-Sen University, Guangzhou, China
\end{abstract}

\section{Key Words}

Rapamycin •Akt/mTORC2 $・$ IL-15 $・$ IGF-1 $・$ Wound healing

\begin{abstract}
Backgroud/Aims: The effects of rapamycin (RPM) on wound healing have been previously studied. However, reciprocal contradictory data have been reported, and the underlying mechanism remains unclear. This study aims to uncover differential role of RPM in regulation of wound healing and explore the possible mechanism. Methods: C57BL/6J mice and epidermal cells were treated with different doses of RPM. The wound re-epithelialization was observed by hematoxylin and eosin (HE) staining. The expression of IL-15 and IGF-1 were detected by immunohistochemistry and quantitative real-time PCR. Epidermal cell survival was determined by CCK- 8 assays. Moreover, the mTORC1 and mTORC2 pathway were examined by western blot analysis. Results: This study showed that differential doses of RPM could lead to separate consequences in epidermis. Histological analyses showed that low-dose RPM promoted wound healing, and enhanced the expression of IL-15 and IGF-1. Futhermore, western blot analysis showed that the effect of low-dose RPM in epidermis were not through mTORC1 pathway. Instead, activation of the Akt/mTORC2 pathway was involved in low-dose RPM-induced IL-15 and IGF-1 production in epidermis, while high-dose RPM inhibited the expression of IL-15 and IGF-1 and the activity of MTORC1 and mTORC2 pathway. Conclusion: This study for the first time demonstrated that RPM-mediated wound healing was dose-dependent.
\end{abstract}

(C) 2017 The Author(s)

Published by S. Karger AG, Basel

\section{Introduction}

Rapamycin (RPM) is usually administered for prophylactic control of acute rejection of transplanted solid organs as well as for coating arterial stents and inhibiting tumor cell growth and tumor angiogenesis [1-5]. Hence, it is important in oncology, cardiology, and 


\section{Cellular Physiology Cell Physiol Biochem 2017;42:1755-1768

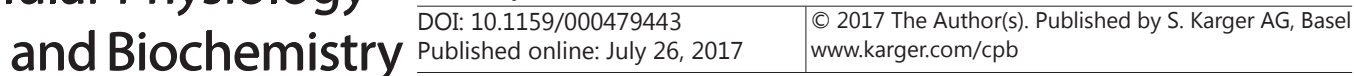 \\ Bai et al.: Differential Role of RPM in Wound Healing}

transplantation medicine [6]. Moreover, the effects of RPM on wound healing have been extensively studied [7-9]. However, there has been contradictory information regarding whether RPM delays wound healing via a $\gamma \delta$ T cell defect in the skin tissue as reported by Robyn E. Mills [1] or promotes wound healing via RPM-induced autophagy in the epidermis, thus leading to faster re-epithelialization and stopping the progression of burn depth in a rat model, as described by Xiao et al. [10].

IL-15 secreted by keratinocytes is required for dendritic epidermal T cell (DETC) development and epidermal homeostasis $[11,12]$. DETCs are the exclusive producer of insulin-like growth factor-1 (IGF-1) in the epidermis [13-15]. For patients with serious skin defects, DETCs respond to damage-induced IL-15, and then DETCs produce IGF-1, which plays an important role in maintaining the skin barrier function and accelerating the wound repair process [13-15]. However, whether RPM affects the secretion of IL-15 and IGF-1 in epidermis during wound repair remains unclear.

It is well known that RPM inhibits the serine/threonine kinase mammalian target mTOR. mTOR is formed by two separate protein complexes, mTOR complex 1 (mTORC1) and mTOR complex 2 (mTORC2), and RPM has generally been believed to interact with mTORC1 but not mTORC2 $[1,16,17]$. However, prolonged treatment of some cell lines with RPM results in reduced levels of mTORC2, as well as decreased phosphorylation of Akt Serine 473, the downstream target of mTORC2 $[6,18,19]$. Interestingly, short-term treatment resulted in increased Akt phosphorylation due to compensatory, high levels of mTORC2 [18]. Previous work has shown that responses of Akt in tumor cells to RPM may be dose-related [17, 19]. In addition, at lower RPM doses, researchers found elevated levels of phosphorylated Akt (Ser473), and when the concentration of RPM increased, the level of phosphorylated Akt (Ser473) decreased.

Recently, several studies have shown that IL-15 can augment the activation of the mTOR pathway. Consistently, suppression of the mTOR pathway may abolish the IL-15-induced advantageous activity in cells $[20,21]$. Moreover, high-dose and prolonged treatment with RPM, which impairs the mTOR pathway, remarkably reduces IGF-1 secretion from DETCs [1]. Above all, how RPM interacts with Akt and mTORC2 remains unclear. Furthermore, the roles of RPM in regulation of IL-15, IGF-1 and mTOR in the wound healing process remain unanswered.

In the presentstudy, we show for the first time thatlow-dose RPM enhances the expression of IL-15 and IGF-1 in the epidermis, and promotes wound healing. More importantly and interestingly, we uncover that low-dose RPM activates phosphorylation of SIN1 at Threonine 86 in epidermal cells, enhancing activation of the Akt/mTORC2 pathway. These findings may help us better understand the RPM-mediated regulation of wound healing.

\section{Materials and Methods}

Animals

C57BL/6J (C57) mice (male, 20-22 g) at 8 to 10 weeks of age were purchased from the Experimental Animal Department of the Third Military Medical University. All animals were maintained under specific pathogen-free conditions. All animal studies were performed in compliance with the guidelines the Ethic Committee for Animal Experiment of The Third Military Medical University.

\section{Preparation and administration of rapamycin}

A stock powder of RPM (25 mg, Selleck Chemicals, USA) was dissolved in dimethyl sulfoxide at a final concentration of $20 \mu \mathrm{g} / \mu \mathrm{L}$. This RPM solution was then divided into aliquots of $10 \mu \mathrm{l}$ each and stored at $-80^{\circ} \mathrm{C}$.

Epidermal cells were treated with different concentrations of RPM. Doses of $5 \mathrm{ng} / \mathrm{ml}$ RPM or $20 \mathrm{ng} /$ $\mathrm{ml}$ RPM served as the low dose and high dose, respectively, for cell treatment administration in vitro. The doses were chosen based on our pilot experiments (for all online suppl. material, see www.karger.com/ doi/10.1159/000479443, Supplementary Fig. S4). 


\section{Cellular Physiology Cell Physiol Biochem 2017;42:1755-1768 \begin{tabular}{l|l} 
and Biochemistry Published online:July 26, 2017 & $\begin{array}{l}\text { (c) } 2017 \text { The Author(s). Published by S. Karger AG, Basel } \\
\text { www.karger.com/cpb }\end{array}$ \\
\hline
\end{tabular}}

Bai et al.: Differential Role of RPM in Wound Healing

C57 mice were injected i.p. daily with $200 \mu \mathrm{l}$ of $0.2 \%$ carboxymethyl cellulose (CMC, Kermel, CHN) and $0.25 \%$ Tween 80 (Sigma-Aldrich, GER) in distilled $\mathrm{H}_{2} \mathrm{O}$ containing $0.04 \mathrm{mg} / \mathrm{kg} \mathrm{RPM} \mathrm{or} 4 \mathrm{mg} / \mathrm{kg} \mathrm{RPM}$, serving as the low dose and high dose. The doses were chosen based on the manufacturer's specifications (Selleck Chemicals, USA) and our pilot experiments (see supplementary material, Supplementary Fig. S5).

\section{Isolation of epidermal cells}

Skin harvested from C57 mice (male, 20-22 g) at 8 to 10 weeks of age were washed with sterile phosphate-buffered saline (PBS, pH 7.4) twice. Then, the skin sample was cut into approximately $0.5 \mathrm{~cm}^{2}$ pieces and digested with $0.5 \mathrm{~g} / \mathrm{l}$ Dispase II (Sigma-Aldrich, GER) at $4{ }^{\circ} \mathrm{C}$ overnight. After that, the epidermis was separated from the dermis. The epidermis was washed with PBS, collected and minced, then digested with $0.5 \%$ trypsin at $37^{\circ} \mathrm{C}$. After 10 minutes, Roswell Park Memorial Institute-1640 (RPMI-1640, Gibco, USA) culture medium containing $10 \%$ fetal bovine serum was added to discontinue the digestion. After being filtered through a cell strainer, the cells were collected via centrifugation. The cells were cultured in RPMI 1640 medium supplemented with 10\% fetal bovine serum, $100 \mathrm{mg} / \mathrm{ml}$ of streptomycin, $100 \mathrm{U} / \mathrm{ml}$ penicillin and $2 \mathrm{mM}$ glutamine (HyClone, USA) and used for the following experiments.

\section{Cell Counting Kit-8 (CCK-8) assay}

An epidermal cell suspension was adjusted to $1 \times 10^{4} / \mathrm{ml}$, and $100 \mu \mathrm{l}$ of the cell suspension was seeded in each well of a 96-well plate. Either low-dose or high-dose RPM was added to each corresponding well. Cells cultured without RPM served as controls. The cells were cultured at $37^{\circ} \mathrm{C}$ in a $5 \% \mathrm{CO}_{2}$ incubator. After culture for 12 hours or 24 hours, $10 \mu \mathrm{l}$ of CCK-8 reagent (Dojindo, JPN) was added to each well. After incubation at $37^{\circ} \mathrm{C}$ for 2 hours, the absorbance was measured at $450 \mathrm{~nm}$ using an enzyme-linked immunosorbent assay reader (Thermo Varioskan Flash, USA) as previously reported [22]. Experiments were repeated 5 times.

\section{Quantitative real-time PCR}

An epidermal cell suspension was adjusted to $1 \times 10^{6} / \mathrm{ml}$, and $1 \mathrm{ml}$ of the cell suspension was seeded in each well of a 12-well plate. The epidermal cells were isolated from the epidermis of C57 adult mice and were cultured in RPMI 1640 medium supplemented with 10\% fetal bovine serum for 2 hours. After treatment with either $5 \mathrm{ng} / \mathrm{ml} \mathrm{RPM} \mathrm{(low} \mathrm{dose)} \mathrm{or} 20 \mathrm{ng} / \mathrm{ml}$ RPM (high dose) for 24 hours in 12-well plates, cultured cells were washed with PBS, and total RNA was extracted with an RNeasy ${ }^{\circledR}$ Mini kit (QIAGEN, GER) according to the manufacturer's instructions. The RNA concentration and quality were measured using a DU800 UV/Vis spectrophotometer (Beckman Coulter, USA), and mRNA was reverse transcribed using First Strand cDNA Synthesis kits (TOYOBO, JPN). Real-time PCR was performed using SYBR Green PCR Master Mix (TOYOBO, JPN) under the following conditions: $95^{\circ} \mathrm{C}$ for 2 minutes followed by 50 cycles of $95^{\circ} \mathrm{C}$ for 15 seconds, $60^{\circ} \mathrm{C}$ for 15 seconds, and $72^{\circ} \mathrm{C}$ for 32 seconds. mRNA levels were quantified using an ABI PRISM 7500 Sequence Detection System (Applied Biosystems, Foster City, CA) according to the manufacturer's protocols. The data were analyzed by the $2^{-\Delta \Delta}$ threshold $(\mathrm{Ct})$ method, and GAPDH served as an internal control. The sequences of primers used were as follows:

IL-15:5'-GGATTTACCGTGGCTTTGAGTAATGAG-3' (forward),5'-GAATCAATTGCAATCAAGAAGTG3 ' (reverse );IGF-1:5' - GGACCAAGGGGCTT TTACTT-3' (forward), 5 ' - GCAACACTCATCC ACAATG3'(reverse);GAPDH:5'-CGTGCCGCCTGGAGAAAC-3'(forward),5'-AGTGGGAGTTGCTGTTGAAGTC-3' (reverse).

\section{Western blot analysis}

After treatment of cells with different concentrations of RPM in vitro or in vivo, proteins were extracted from cells using lysis kits (KeyGEN BioTECH, CA) according to the manufacturer's protocol. The cell lysates were agitated for 20 minutes followed by centrifugation at $14,000 \mathrm{rpm} / \mathrm{min}$ for 15 minutes at $4^{\circ} \mathrm{C}$. The supernatant was collected as total cellular protein. Protein concentrations were determined using a BCA protein assay (Thermo Scientific, USA). An equal amount of protein $(20 \mu \mathrm{g})$ from each sample was loaded onto $10 \%$ SDS-PAGE gels, and electrophoresis was performed at 80 volts for 30 minutes and then at 100 volts for 1.5 hours. The separated proteins were then transferred to a polyvinylidenedifluoride (PVDF) membrane (Millipore Immobilon, USA). The PVDF membrane was blocked with Tris-buffered saline (TBS) containing 3\% bovine serum albumin (BIOSHARP, CA) for 2 hours at room temperature and then incubated with primary antibodies against IGF-1 and IL-15 (1:200, Santa Cruz Biotechnology, USA); p-p70S6K (Thr389), S6K, p-Akt (Ser473), Akt, p-4E-BP1 (Thr37/46), 4E-BP1 and p-SIN1(Thr86) (1:1,000, Cell Signaling Technologies, Beverly, USA); SIN-1, mTOR, Raptor and Rictor (1:1,000, Abcam, UK); and GAPDH

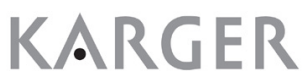




\section{Cellular Physiology Cell Physiol Biochem 2017;42:1755-1768

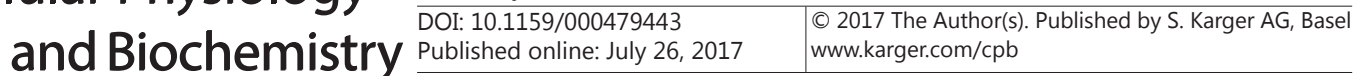 \\ Bai et al.: Differential Role of RPM in Wound Healing}

(1:5000, KANGCHEN BIO-TECH, CA) at $4^{\circ} \mathrm{C}$ overnight. The membranes were subsequently washed 5 times with TBS containing $0.1 \%$ Tween-20 (TBST) and then incubated with HRP-labeled secondary antibody (1:5000, ZSGB-BIO, CHN) for 1 hour at room temperature. The membranes were washed with TBST again and finally visualized and analyzed using enhanced chemiluminescence (Pierce, USA) and a ChemiDoc ${ }^{\mathrm{TM}}$ XRS western blot detection system (Bio-Rad, USA).

\section{Wounding procedure}

For RPM administration, C57 mice were injected i.p. daily with $200 \mu \mathrm{l}$ of low-dose RPM (0.04 mg/kg), high-dose RPM (4 mg/kg) or vehicle control (dissolved in $0.2 \%$ CMC and $0.25 \%$ Tween 80 in distilled $\mathrm{H}_{2} \mathrm{O}$ ) for 15 days before wounding, and daily administration was continued during the wound healing process (on post-wounding days 0-7). The administration dose of RPM was chosen based on our pilot study (see supplementary material, Supplementary Fig. S5).

Wounding was performed on mice anesthetized with $1 \%$ sodium pentobarbital. Briefly, the dorsal surface of the mouse was shaved, and two sets of sterile full-thickness wounds were generated using a sterile 4-mm punch tool. The survey of wound healing was performed as previously reported with minor revision $[23,24]$. Wounds were left uncovered, and mice were kept individually with sterile paper bedding. Wounds on at least five mice were examined in each group in three independent experiments.

Analysis of the re-epithelialization during wound healing

On day 3 and day 6 post-wounding, mice were sacrificed and the wound tissues were carefully biopsied. Tissues were fixed with $4 \%$ formaldehyde, embedded in paraffin, and sectioned at a thickness of $5 \mu \mathrm{m}$. Hematoxylin and eosin (HE) staining and histological analyses were performed, and the length of the neo-epithelium was determined using IPP 6.0 software; the measurement procedures were performed blindly by two pathologists $[23,24]$. Experiments were repeated 5 times.

\section{Immunohistochemistry}

The paraffin sections were deparaffinized and rehydrated. After incubation with Antigen Retrieval Solution (Boster Biotechnology, CHN) for 20 minutes, the sections were incubated with $3 \% \mathrm{H}_{2} \mathrm{O}_{2}$ for 15 minutes, blocked with 10\% normal goat serum (Zhongshan Biology Company, CHN) for 1 hour at $37^{\circ} \mathrm{C}$ and then incubated with primary antibody (IGF-1, 1:200, Santa Cruz Biotechnology, USA; IL 15, 1:200, Santa Cruz Biotechnology, USA) overnight at $4^{\circ} \mathrm{C}$. Sections were incubated with biotinylated goat-antirabbit IgG antibody (Zhongshan Biology Company, CHN) for 15 minutes and sequentially incubated with avidin peroxidase reagent (Zhongshan Biology Company, CHN). Diaminobenzidine solution was used as the chromogenic agent. After counterstaining with hematoxylin, sections were photographed using an optical microscope (CTR6000, Leica, GER) [24].

\section{Statistical analysis}

Statistical comparisons were performed using Student's $t$-test or one-way ANOVA with Graphpad Prism 6.0 software (Graphpad Software Inc, USA). Data are presented as the mean \pm standard deviation (SD). In all cases, a p value less than 0.05 was considered statistically significant.

\section{Results}

Epidermal cell survival was promoted in the low-dose RPM group compared with the control or high-dose RPM groups

Epidermal cell survival in vitro under the condition of different concentrations of RPM was determined using a CCK-8 assay (Fig. 1A). After 12 hours of culture, the average of the absorbance was 0.782 in the low-dose RPM group ( $5 \mathrm{ng} / \mathrm{ml}$ ), which was higher than that in the high-dose RPM group (20 ng/ml, 0.644) and the control group (0.676) (Fig. 1A), indicating that more cells were viable in the low-dose RPM group than in the other two groups. After culture for 24 hours, the highest number of viable cells was found in the lowdose RPM group, followed by the control group, and the lowest number of cells was found in the high-dose RPM group (the average absorbance was $0.77,0.684$ and 0.562 , in sequence; Fig. 1A).

\section{KARGER}




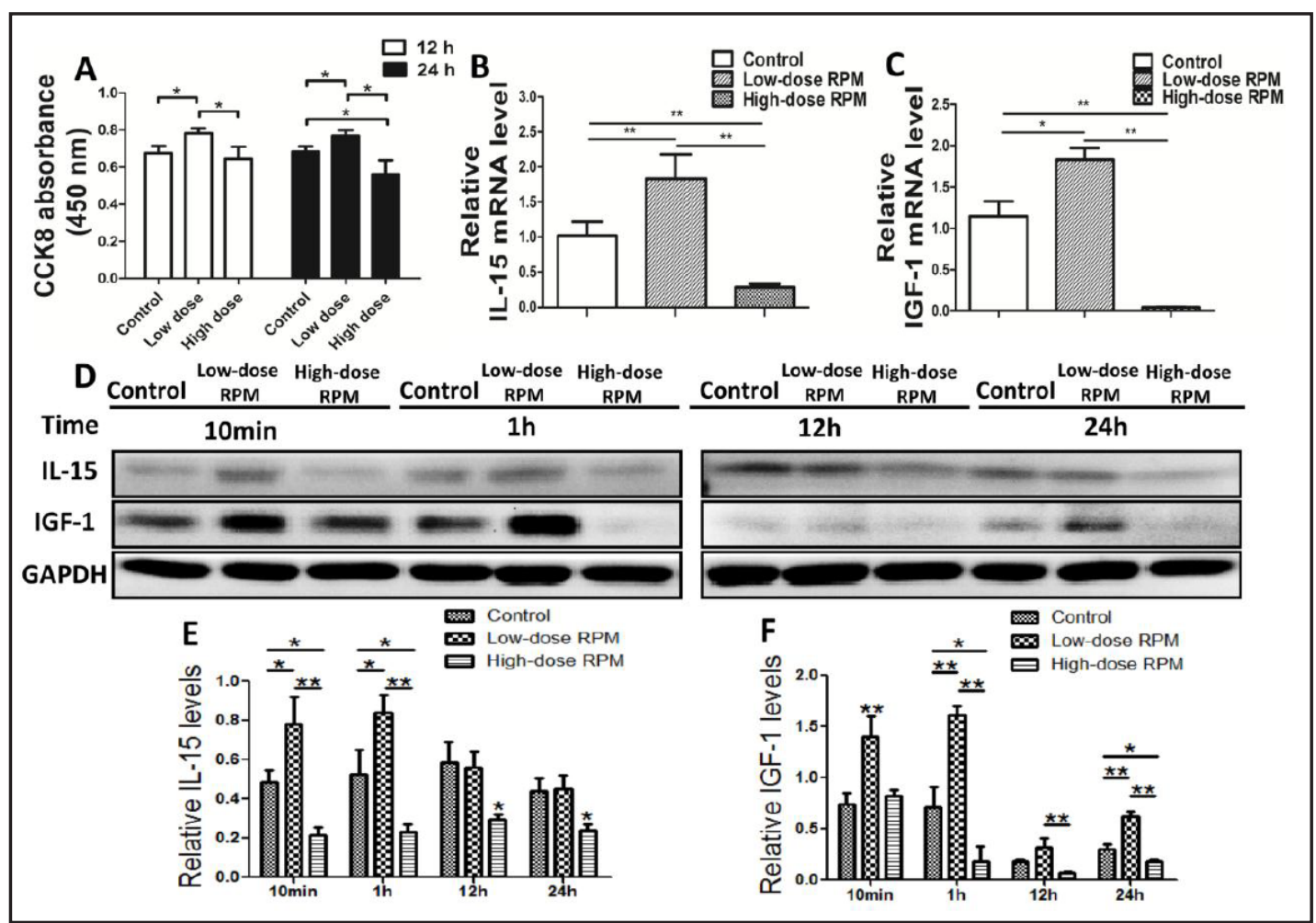

Fig. 1. Low-dose RPM enhances the expression of IL-15 and IGF-1 in epidermal cells. (A) The number of viable epidermal cells was determined with CCK-8 assays. Cells were treated with low-dose RPM (5 ng/ml), high-dose RPM (20 ng/ml) and vehicle control for 12 and 24 hours. (B, C) The mRNA level of IL-15 and IGF1 were determined in epidermal cells treated with low-dose RPM, high-dose RPM and vehicle control using real-time PCR. (D) Western blot analysis demonstrating the protein levels of IL-15 and IGF-1 in epidermal cells treated with low-dose RPM, high-dose RPM and vehicle for the indicated time. The relative densities of the (E) IL-15 and (F) IGF-1 protein level in each group are shown. Data are shown as the mean \pm SD of 3-5 independent experiments; ${ }^{* *} \mathrm{p}<0.01 ;{ }^{*} \mathrm{p}<0.05$.

\section{Expression of IL-15 and IGF-1 were enhanced in the presence of low-dose RPM}

The expression of IL-15 and IGF-1 was detected using real-time PCR and Western blot. As shown, the IL-15 (Fig. 1B) and IGF-1 (Fig. 1C) mRNA level were elevated in the low-dose RPM group compared with the control group. High-dose RPM in fact significantly inhibited the expression of IL-15 (Fig. 1B) and IGF-1 (Fig. 1C). We further detected the protein level of IL-15 and IGF-1 at different RPM treatment times. The IL-15 protein level was found to be rapidly upregulated 10 minutes after treatment with low-dose RPM. In contrast, IL15 production was significantly attenuated by high-dose RPM (Fig. 1D and 1E). The IGF-1 protein level in epidermal cells was also upregulated after low-dose RPM treatment for 10 minutes, while high-dose RPM inhibited the expression of IGF-1 after the epidermal cells were treated for 1 hour (Fig. 1D and 1F).

As IGF-1 is produced exclusively by DETCs in the epidermal compartment [14], we therefore detected the number of DETCs in the control, low-dose RPM and high-dose RPM groups (see supplementary material, Supplementary Fig. S1A and S1B). It was found that the number of DETCs was not significantly different in the low-dose RPM group compared with the vehicle control after 24 hours, and was apparently decreased in the high-dose RPM group in vitro. $\gamma \delta$ TCR and IGF-1 were further co-stained to evaluate the production of IGF-1 in the DETCS using FACS (see supplementary material, Supplementary Fig. S2). The DETCderived IGF-1 was significantly increased in epidermal cells treated with both recombinant IL-15 (100 ng/ml) and low-dose RPM (see supplementary material, Supplementary Fig. S2E) compared with cells treated with either recombinant IL-15 (see supplementary material, 
Fig. 2. Low-dose RPM potentiates mTORC2 pathway activation in epidermal cells at different time points. (A) Primary epidermal cells were treated with low-dose RPM (5 $\mathrm{ng} / \mathrm{ml}$ ), high-dose RPM (20 ng/ml) and vehicle control for the indicated period, followed by western blot analysis. The relative densities of the (B) mTOR, (C) Rictor, (D) p-SIN1(Thr86), Akt protein levels in each group are shown. Data are shown as the mean \pm SD of 5 independent experiments; ** $\mathrm{p}<0.01 ;{ }^{*} \mathrm{p}<$ 0.05 . (E) SIN-1, (F) p-Akt (Ser473) and (G)

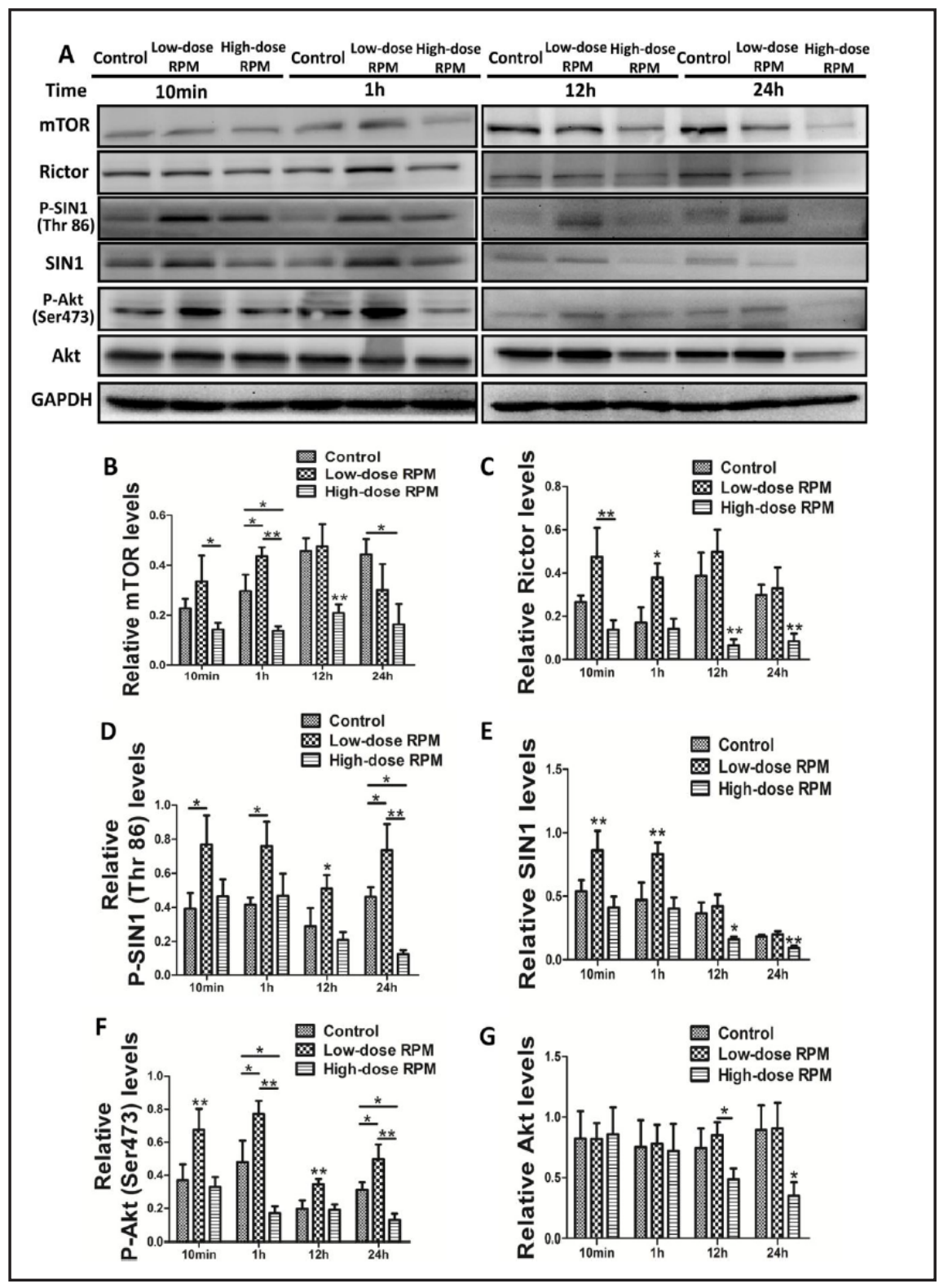

Supplementary Fig. S2D) or low-dose RPM (see supplementary material, Supplementary Fig. S2B) alone and was almost abrogated in the high-dose RPM group (see supplementary material, Supplementary Fig. S2C). In addition, treatment with recombinant IL-15 decreased the reduction of IGF-1 in the high-dose RPM group (see supplementary material, Supplementary Fig. S2F).

mTORC2 but not mTORC1 in epidermal cells was activated in the presence of low-dose $R P M$

After stimulation with low-dose RPM, the protein levels of mTOR were upregulated, especially at 12 hours (Fig. 2A and 2B). Meanwhile, the protein expression of Rictor, p-SIN1 (Thr86) and SIN1 were also upregulated after the cells were treated with low-dose RPM (Fig. 2A, and 2C-2E), while the expression of Rictor, p-SIN1 (Thr86) and SIN1 were inhibited by high-dose RPM at 12 or 24 hours (Fig. 2A, and 2C-2E). The data also showed that the downstream p-Akt (Ser473) protein level was upregulated rapidly 10 min after administration of low-dose RPM and reached the highest level in 1 hour, whereas high-dose RPM down-regulated the p-Akt (Ser473) level (Fig. 2A, and 2F-2G). 
Fig. 3. Low-dose RPM does not affect mTORC1 in epidermal cells at different time points. (A) Primary epidermal cells were treated with low-dose RPM (5 ng/ $\mathrm{ml}$ ), high-dose RPM (20 ng/ml) and vehicle control for the indicated period, followed by western blot analysis. The relative densities of (B) Raptor, (C) p-4E-BP1 (Thr37/46), (D) 4E-BP1, p - p 70 S 6 K ( Thr389) and (F) p70S6K protein levels in each group are shown. Data are shown as the mean \pm SD of 5 independent experiments; ${ }^{* *} \mathrm{p}<0.01$; $* \mathrm{p}<0.05$.
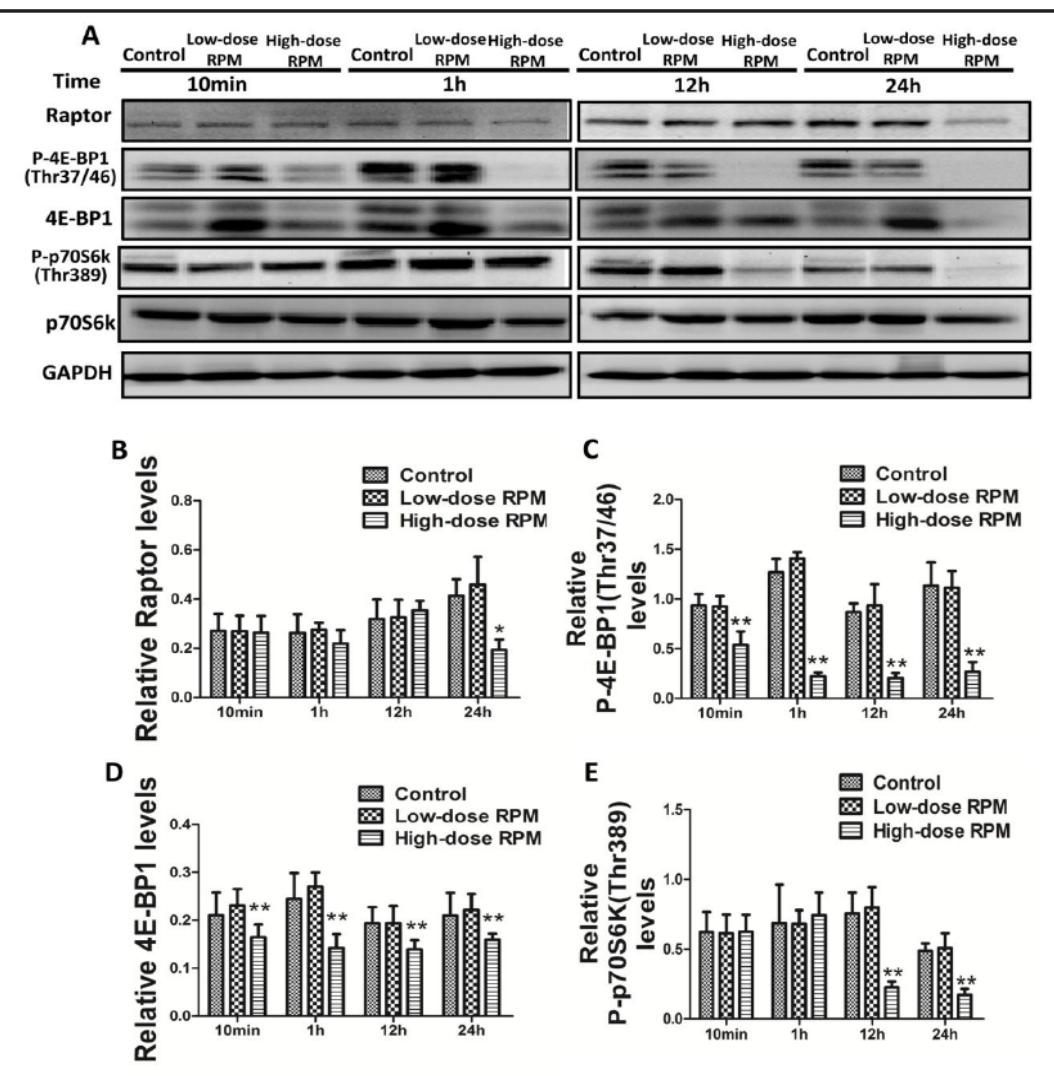

F

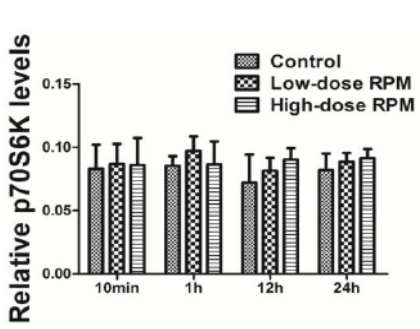

There was no significant difference in Raptor, p-4E-BP1 (Thr37/46) and p-p70S6K (Thr389) protein levels between the low-dose RPM group and the control group (Fig. 3A-3C and 3E). However, high-dose RPM significantly inhibited the expression of Raptor, p-4E-BP1 (Thr37/46) and p-p70S6K (Thr389) at 12 or 24 hours (Fig. 3A-3C and 3E). In addition, 4EBP1 (Fig. 3A and 3D) and p70S6K are also shown (Fig. 3A and 3F).

\section{Wound healing was accelerated after treatment with low-dose RPM}

As the different doses of RPM showed differential effects on epidermal cell proliferation, we then tested whether either low- or high-dose RPM could also have differential role in epithelialization during wound healing from day 0 to day 7 . The results showed that administration of high-dose RPM to mice delayed wound healing compared with the control group (Fig. 4A and 4B), while administration of low-dose RPM to mice significantly promoted wound healing on days 3, 4 and 6 post-wounding (Fig. 4A and 4B). The average healing time in the low-dose RPM group was 6 days, and wounds in the control and high-dose RPM groups remained open after 6 days (Fig. 4A and 4B).

We then tested the speed of re-epithelialization, i.e., the measurement of the length of the newly formed epithelium on wounds, under either low- or high-dose RPM treatment. The results showed that the average length of the epithelial tongue was $901 \mu \mathrm{m}$ in the lowdose RPM group, $619 \mu \mathrm{m}$ in the control group, and $340 \mu \mathrm{m}$ in the high-dose RPM group at 


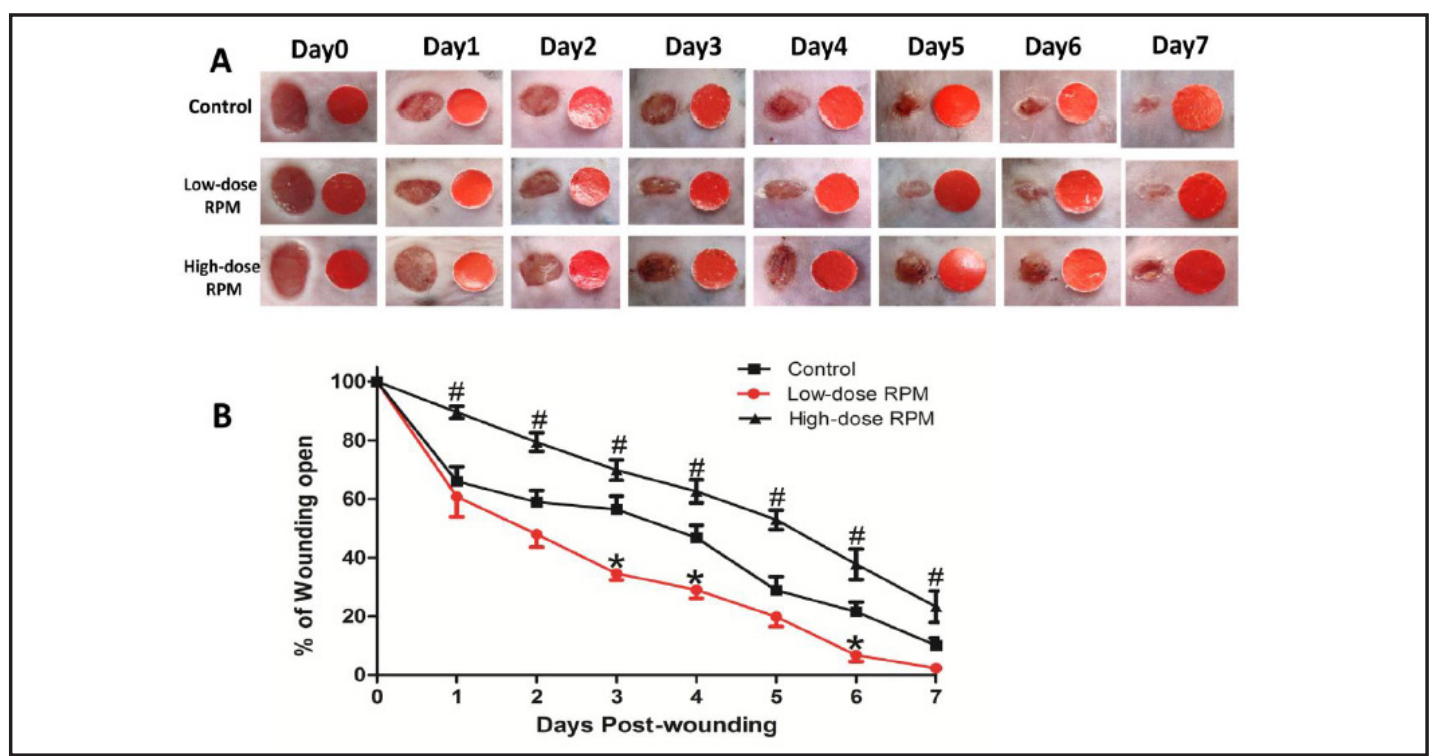

Fig. 4. Low-dose RPM promotes wound healing. (A) The macroscopic appearance of the wounds postsurgery on the low-dose RPM $(0.04 \mathrm{mg} / \mathrm{kg})$, high-dose RPM $(4 \mathrm{mg} / \mathrm{kg})$ and vehicle control mice on day 0 to day 7. (B) The ratio of the left wound in every group at different time points from day 0 to day 7. Data are presented as the mean \pm SD and are representative of three experiments. *less than others, $p<0.05$; "greater than others, $\mathrm{p}<0.05$. At least five mice per condition were examined in each experiment.

Fig. 5. Low-dose RPM promotes re-epithelialization of the wound. (A) The newly formed epithelium in the wound tissue at day 3 and day 6 post-wounding in different groups using H\&E staining, and (B) the length of in the three groups at days 3 and 6. The values were calculated as the mean $\pm \mathrm{SD}(\mathrm{n}=5)$; ${ }^{* *} \mathrm{p}<0.01 ;{ }^{*} \mathrm{p}<0.05$. the epithelium tongue

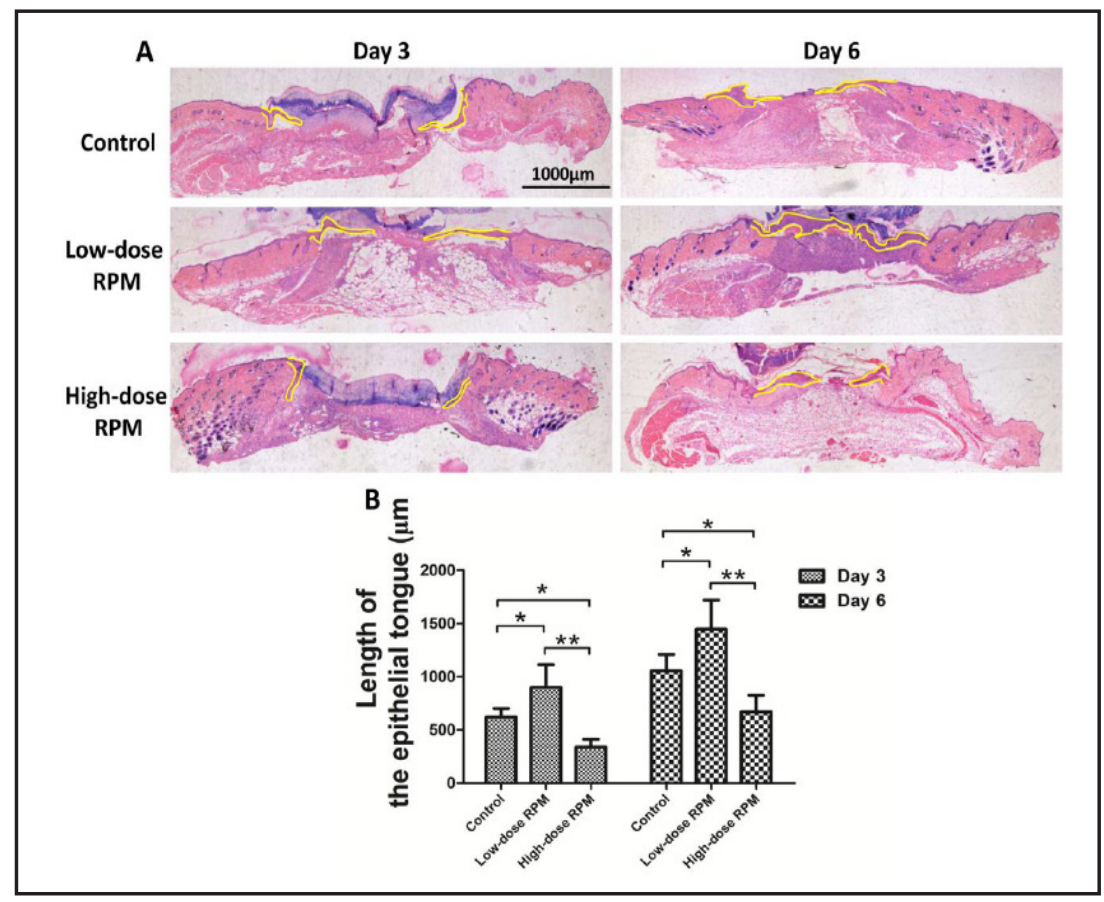

day 3 (Fig. 5A and 5B). At day 6, the average length of the epithelial tongue was $1449 \mu \mathrm{m}$ in the low-dose RPM group, $1058 \mu \mathrm{m}$ in the control group, and $672 \mu \mathrm{m}$ in the high-dose RPM group (Fig. 5A and 5B).

Differential effect of RPM on the number of DETCs around the wound site

We further determined the number of DETCs around the wound site in vivo under the different doses of RPM. It was found that the average ratios of DETCs/total epidermal cells were $4.9 \%$ in the low-dose RPM group and $5.9 \%$ in the control group, but this difference was 
Fig. 6. Low-dose RPM enhances the expression of IL-15 and IGF-1 around the wound edge. (A, D) IL-15, IGF-1 and GAPDH protein levels were determined using Western blot, and the relative densities of the (B) IL-15 and (E) IGF-1 protein level in each group are shown. The values were calculated as the mean \pm SD (n = 5); ${ }^{* *} \mathrm{p}<0.01 ;{ }^{*} \mathrm{p}<0.05$. (C, F) IL-15 and IGF-1 positive areas around the wound edge on day 3 and day 6 post-wounding using immunohistochemistry.

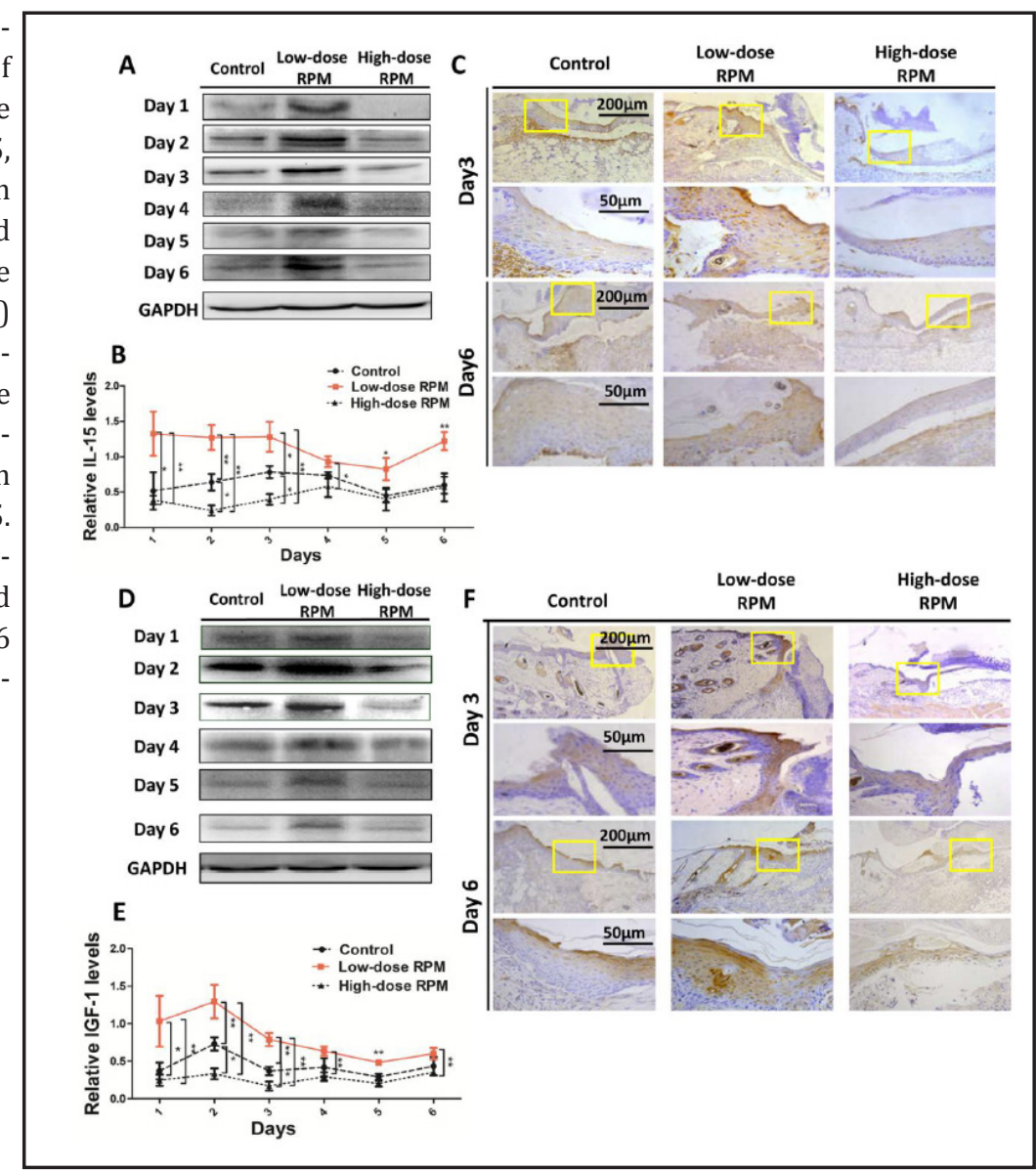

not statistically significant. There were fewer DETCs (1.87\%) around the wound in high-dose RPM-treated mice compared with wounds of mice treated with low-dose RPM and vehicle control (see supplementary material, Supplementary Fig. S3A and S3B).

Differential effect of RPM on IL-15 and IGF-1 expression around the wound site

The expression of IL-15 and IGF-1 around the wound site was detected from day 1 to day 6 post-wounding after administration of different doses RPM (Fig. 6). It was found that the IL-15 (Fig. 6A and 6B) and IGF-1 (Fig. 6D and 6E) protein levels were upregulated in the lowdose RPM group and reached the highest level on day 1 to day 3 after RPM administration based on a Western blot analysis, while high-dose RPM significantly inhibited the expression of IL-15 (Fig. 6A and 6B) and IGF-1 (Fig. 6D and 6E). Consistent with the Western blot results, immunohistochemical staining for IL-15 (Fig. 6C) and IGF-1 (Fig. 6F) was found to be more intensive in the low-dose RPM group than in the high-dose group.

\section{Differential effect of RPM on the phosphorylation of Akt Ser473 in vivo}

In the mouse wound healing model, we determined the effects of different doses of RPM on mTOR signaling during the post-wounding from day 1 to day 6 (Fig. 7). Upon low-dose RPM treatment, Akt is phosphorylated at Ser473 from day 1 to day 4 post-wounding. Among the three groups, the p-Akt (Ser473) protein level was highest in the low-dose RPM group. In contrast, the phosphorylation of Akt was severely impaired after treatment with high-dose RPM (Fig. 7A-7C).

Meanwhile, signaling through mTORC1 was not affected by different doses of RPM compared with vehicle control (Fig. 7A and 7D-G). The protein expression of p-p70S6k (Thr389) (Fig. 7A and 7D) and p-4E-BP1 (Thr37/46) (Fig. 7A and 7F) in the low-dose RPM group was similar to that in the control group. However, phosphorylation of p70S6k 


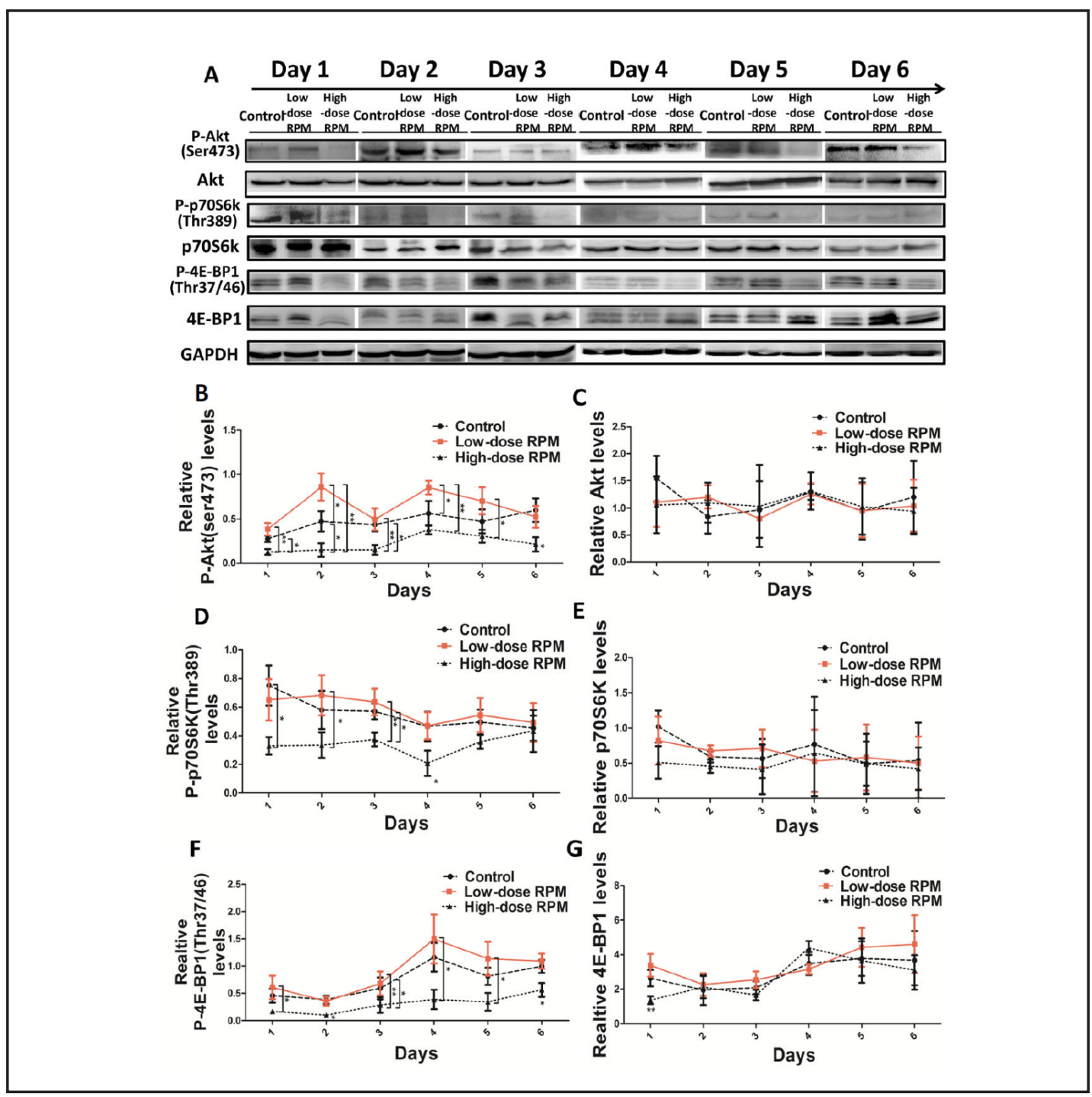

Fig. 7. Low-dose RPM activates the mTORC2 but not mTORC1 pathway in the epidermis around the wound edge. (A) C57 mice were treated with low-dose RPM $(0.04 \mathrm{mg} / \mathrm{kg})$, high-dose RPM (4 mg/kg) and vehicle control for the indicated period, followed by a western blot analysis. The relative densities of the (B) p-Akt (Ser473), (C) Akt, (D) p-p70S6K (Thr389), (E) p70S6K, (F) p-4E-BP1 (Thr37/46) and (G) 4E-BP1 protein levels in each group are shown. The values were calculated as the mean \pm SD $(n=5) ;{ }^{* *} p<0.01 ;{ }^{*} p<0.05$.

(Thr389) (Fig. 7A and 7D) and 4E-BP1 (Thr37/46) (Fig. 7A and 7F) was repressed in the presence of high-dose RPM.

\section{Discussion}

Rapamycin (RPM), a specific inhibitor of mechanistic target of rapamycin (mTOR), has been shown to have potent immunosuppressive properties and antitumor activity $[1,25]$. It is believed that RPM can inhibit T and B cell activation and proliferation [26]. However, it has also been reported that $\mathrm{T}$ cell proliferation is not affected when treated with RPM at an appropriate concentration [27]. Meanwhile, it has also been reported that RPM can protect neuronal cells from apoptosis by inducing autophagy, which indicates that RPM has a cytoprotective effect $[28,29]$.

\section{KARGER}


The effects of RPM on wound healing have been studied and the results are paradoxical. Mills has reported that i.p. injection of approximately $100 \mathrm{mg} / \mathrm{kg}$ RPM in adult C57 mice was responsible for a delay in wound closure by impairing the function of dendritic epidermal $\mathrm{T}$ cells (DETCs), including reducing DETC-derived factors such as insulin-like growth factor-1 (IGF-1) [1]. However, Xiao found that i.p. injection of $1 \mathrm{mg} / \mathrm{kg}$ RPM could ameliorate the early progression of burn depth and promote wound healing in a rat model of deep seconddegree burn wounds through autophagy enhancement [10]. Given these studies, we sought to determine if differential doses of RPM would lead to separate consequences in wound healing.

To test this hypothesis, we cultured epidermal cells isolated from adult C57 mice, treated with different concentration of RPM for 12 hours and 24 hours. According to the CCK- 8 assay, our results revealed that a higher number of viable epidermal cells were present in the lowdose RPM group (5 ng/ml) than in the control and high-dose RPM groups $(20 \mathrm{ng} / \mathrm{ml}$ ) at 12 hours and 24 hours. Until 24 hours of treatment, epidermal cell numbers decreased in the high-dose RPM group compared with the other two groups (Fig. 1A). These results indicated that (i) low-dose RPM had a cytoprotective effect that promoted cell survival in vitro (obviously from 12 hours to 24 hours), and (ii) prolonged treatment ( 24 hours) with high-dose RPM inhibited cell survival and cell proliferation.

Based on the following tests, we found that IL-15 was elevated in the presence of lowdose RPM in epidermal cells at both the mRNA and protein level. Previous studies have verified that keratinocytes are the main component and the major source of IL-15 in the epidermis [30], and IL-15 augmented mTOR signaling, which is related to cell metabolism and respiration. Lee and colleagues reported that IL-15 could activate PI3K and phosphorylate Akt at Thr308 [31], and furthermore, activation of PI3K/Akt (Thr308) phosphorylated SIN1 at Thr86, enhancing mTORC2 kinase activity, which leads to phosphorylation of Akt Ser473 by mTORC2, thereby catalyzing full activation of Akt [32]. In our study, we found that the expression of p-SIN1 (Thr86), Rictor and downstream p-Akt (Ser473) was enhanced under the condition of low-dose RPM, which indicated that mTORC2 signaling was activated due to the increase in IL-15 induced by low-dose RPM. In addition, activation of mTORC2 has been shown to maintain the survival of keratinocytes [33], which was also confirmed in our study as mentioned above.

It is generally believed that RPM is a specific (mTOR) inhibitor that interacts with mTORC1 (composed of mTOR, mLST8, and Raptor) [1], while the distinct complex of mTOR, mLST8, SIN1 and Rictor (mTORC2) is not thought to be RPM sensitive [6, 34]. However, Sarbassov et al. reported that prolonged RPM treatment inhibits mTORC2 assembly and Akt in cells [6]. In our study, to determine if dose-related RPM can alter the levels of intact mTORC1 and/or mTORC2, we cultured the epidermal cells isolated from adult C57 mice treated with low-dose and high-dose RPM for 10 minutes or 1, 12, or 24 hours (Fig. 2 and Fig. 3). Our experiments demonstrated that after treatment with high-dose RPM for 24 hours, the expression of p-SIN1 (Thr86), p-Akt (Ser473) and Rictor in epidermal cells was drastically decreased. In addition, the phosphorylation of downstream p70S6K (Thr389), 4E-BP1 (Thr37/46) and Raptor was also impaired. These results showed that prolonged treatment (24 hours) with high-dose RPM significantly inhibited mTORC1 and mTORC2 signaling. Interestingly, low-dose RPM treatment did not affect the expression of p-p70S6K (Thr389), 4E-BP1 (Thr37/46) and Raptor compared with vehicle control during the four time points. Instead, p-SIN1 (Thr86) was activated by low-dose RPM compared with vehicle control in 10 minutes, and the downstream target of mTORC2, p-Akt (Ser473), was increased rapidly after 10 minutes as well and reached the highest level in 1 hour. Moreover, the expression of Rictor and mTOR were upregulated after treatment for 12 hours. In addition, Phung reported that p-Akt was elevated at lower rapamycin doses (1-4 ng/ml) in primary endothelial cells isolated from human skin, and it steadily diminished as the concentration of rapamycin was increased [17]. Based on the data here and previous reports, we concluded that RPM might interact with mTORC1 and mTORC2 in a dose- and time-dependent manner, and low-dose RPM treatment resulted in increased levels of mTORC2, as well as increased 


\section{Cellular Physiology Cell Physiol Biochem 2017;42:1755-1768 \\ \begin{tabular}{l|l} 
DOI: 10.1159/000479443 & and Biochemistry \\
Published online: July 26, 2017 & $\begin{array}{l}\text { 2017 The Author(s). Published by S. Karger AG, Basel } \\
\text { www.karger.com/cpb }\end{array}$
\end{tabular} \\ Bai et al.: Differential Role of RPM in Wound Healing}

phosphorylation of the downstream target Akt (Ser473), without affecting the levels of mTORC1. O'Reilly's studies showed that the concentration of $1 \mathrm{nmol} / \mathrm{L}$ RPM in tumor cell culture induces mTORC2 activity, resulting in increased p-Akt (Ser473) levels [19]. Yang Guang and colleagues have revealed that phosphorylation of SIN1 (Thr86) mediated a positive feedback loop between Akt and mTORC2 [32]. Accordingly, enhancement of the activity of the Akt/mTORC2 pathway by low-dose RPM treatment might promote epidermal cell survival.

Notably, IL-15 plays an important role in altering DETC development and homeostasis in epidermis [35, 36]. DETCs are known to play a positive role in wound healing [37], and secretion of insulin-like growth factor-1 (IGF-1) in DETCs plays positive roles in keratinocyte proliferation [13]. In our study, we found that the IL-15 protein level was upregulated, and we further detected the expression of IGF-1 in the presence of low-dose RPM in epidermal cells. Indeed, it was found that the IGF-1 protein level was also upregulated. On the other hand, high-dose RPM inhibited the expression of IGF-1 significantly in epidermal cell culture after 24 hours (Fig. 1). The low-dose RPM-induced upregulation of DETC-derived IGF-1 might also be beneficial to keratinocyte survival and proliferation. Furthermore, we found through a FACS analysis that recombinant IL-15 upregulated the expression of DETC-derived IGF1 in epidermis (see supplementary material, Supplementary Fig. S2). The result revealed that keratinocyte-secreted IL-15 may enhance DETC activation to produce IGF-1 and may mediated a positive feedback loop during wound repair.

The in vitro results inspired us, and we performed wound healing experiments to determine whether the in vitro results were replicable in vivo. It was found that wound healing was accelerated when mice were intraperitoneally injected with low-dose RPM $(0.04 \mathrm{mg} / \mathrm{kg})$ over a prolonged time. Reversely, the same method of prolonged treatment with high-dose RPM (4 mg/kg) hindered the wound healing process (Fig. 4), which has been reported in previous studies $[1,7,9]$.

We found that wound re-epithelialization was enhanced after treatment with low-dose RPM (Fig. 5), and the enhanced wound re-epithelialization might result from the secretion of IL-15 and IGF-1 in the epidermis at the wound edge. In our study, it was found that the expression of both IL-15 and IGF-1 was upregulated at the wound site in the low-dose RPM group and reversely suppressed in the high-dose RPM group (Fig. 6). Thus, upregulated levels of IL-15 might activate DETCs (the number of DETCs in epidermis was not changed in the low-dose RPM group compared with that in the control group (see supplementary material, Supplementary Fig. S3) and more IGF-1 could be secreted from the activated DETCs. In addition, keratinocytes could respond to IGF-1 via IGFR for cell survival and cell proliferation [38], and the enhanced IGF-1 expression might partially explain the accelerated wound healing after the mice were treated with low-dose RPM.

In addition, we detected the expression of the mTOR pathway in epidermis at the wound edge (Fig. 7). It was revealed that the phosphorylation of the downstream target Akt (Ser473) of the mTORC2 pathway was activated when mice were treated with low-dose RPM. At the same time, the expression of p-p70S6K (Thr389) and p-4E-BP1 (Thr37/46) of the mTORC1 pathway was not effected by low-dose RPM treatment. Overall, the in vivo data further confirmed that the Akt/mTORC2 pathway controlled the expression of IL-15 and IGF-1 by regulating the function of epidermal cells, and was involved in the accelerated wound healing process.

In this study, we uncover a novel role of low-dose RPM in regulation of wound healing, i.e., low-dose RPM upregulates the expression of IL-15 and IGF-1 in the epidermis, which maintains the survival of keratinocytes and the activity of DETCs by activating Akt/mTORC2 patway and ultimately accelerating wound healing. Further studies are required to unravel the possible mechanism of how low-dose RPM upregulates the expression of IL-15 and IGF-1. 


\section{Cellular Physiology Cell Physiol Biochem 2017;42:1755-1768 \begin{tabular}{l|l} 
DOI: 10.1159/000479443 & O 2017 The Author(s). Published by S. Karger AG, Basel \\
www.karger.com/cpb
\end{tabular}}

Bai et al.: Differential Role of RPM in Wound Healing

\section{Acknowledgements}

This work was supported by the National Nature Science Foundation of China (NSFC No.81372082 and 81201473), and by a grant from the Southwest Hospital Scientific Research Fund (No. SWH2016JCYB-29).

\section{Disclosure Statement}

The authors of this article declare that there are no conflicts of interest.

\section{References}

1 Mills RE, Taylor KR, Podshivalova K, McKay DB, Jameson JM: Defects in skin gamma delta T cell function contribute to delayed wound repair in rapamycin-treated mice. J Immunol 2008;181:3974-3983.

2 Augustine JJ, Bodziak KA, Hricik DE: Use of sirolimus in solid organ transplantation. Drugs 2007;67:369391.

-3 MacDonald AS: Rapamycin in combination with cyclosporine or tacrolimus in liver, pancreas, and kidney transplantation. Transplant Proc 2003;35:201S-208S.

4 Ruchin PE, Muller DW, Faddy SC, Baron DW, Roy PR, Wilson SH: Long-term clinical follow-up of sirolimuseluting (CYPHER) coronary stents in the treatment of instent restenosis in an unselected population. Heart Lung Circ 2007;16:440-446.

5 Witzig TE, Kaufmann SH: Inhibition of the phosphatidylinositol 3-kinase/mammalian target of rapamycin pathway in hematologic malignancies. Curr Treat Options Oncol 2006;7:285-294.

-6 Sarbassov DD, Ali SM, Sengupta S, Sheen JH, Hsu PP, Bagley AF, Markhard AL, Sabatini DM: Prolonged rapamycin treatment inhibits mTORC2 assembly and Akt/PKB. Mol Cell 2006;22:159-168.

7 Dean PG, Lund WJ, Larson TS, Prieto M, Nyberg SL, Ishitani MB, Kremers WK, Stegall MD: Wound-healing complications after kidney transplantation: a prospective, randomized comparison of sirolimus and tacrolimus. Transplantation 2004;77:1555-1561.

8 Kuppahally S, Al-Khaldi A, Weisshaar D, Valantine HA, Oyer P, Robbins RC, Hunt SA: Wound healing complications with de novo sirolimus versus mycophenolate mofetil-based regimen in cardiac transplant recipients. Am J Transplant 2006;6:986-992.

-9 Schaffer M, Schier R, Napirei M, Michalski S, Traska T, Viebahn R: Sirolimus impairs wound healing. Langenbecks Arch Surg 2007;392:297-303.

10 Xiao M, Li L, Hu Q, Ma L, Liu L, Chu W, Zhang H: Rapamycin reduces burn wound progression by enhancing autophagy in deep second-degree burn in rats. Wound Repair Regen 2013;21:852-859.

11 Edelbaum D, Mohamadzadeh M, Bergstresser PR, Sugamura K, Takashima A: Interleukin (IL)-15 promotes the growth of murine epidermal gamma delta $\mathrm{T}$ cells by a mechanism involving the beta- and gamma c-chains of the IL-2 receptor. J Invest Dermatol 1995;105:837-843.

12 Ye SK, Maki K, Lee HC, Ito A, Kawai K, Suzuki H, Mak TW, Chien Y, Honjo T, Ikuta K: Differential roles of cytokine receptors in the development of epidermal gamma delta T cells. J Immunol 2001;167:1929-1934.

13 Sharp LL, Jameson JM, Cauvi G, Havran WL: Dendritic epidermal T cells regulate skin homeostasis through local production of insulin-like growth factor 1. Nat Immunol 2005;6:73-79.

14 Liu Z, Xu Y, Chen L, Xie J, Tang J, Zhao J, Shu B, Qi S, Chen J, Liang G, Luo G, Wu J, He W, Liu X: Dendritic epidermal T cells facilitate wound healing in diabetic mice. Am J Transl Res 2016;8:2375-2384.

15 Jameson J, Ugarte K, Chen N, Yachi P, Fuchs E, Boismenu R, Havran WL: A role for skin gammadelta T cells in wound repair. Science 2002;296:747-749.

16 Deng Z, Lei X, Zhang X, Zhang H, Liu S, Chen Q, Hu H, Wang X, Ning L, Cao Y, Zhao T, Zhou J, Chen T, Duan E: mTOR signaling promotes stem cell activation via counterbalancing BMP-mediated suppression during hair regeneration. J Mol Cell Biol 2015;7:62-72.

17 Phung TL, Ziv K, Dabydeen D, Eyiah-Mensah G, Riveros M, Perruzzi C, Sun J, Monahan-Earley RA, Shiojima I, Nagy JA, Lin MI, Walsh K, Dvorak AM, Briscoe DM, Neeman M, Sessa WC, Dvorak HF, Benjamin LE: Pathological angiogenesis is induced by sustained Akt signaling and inhibited by rapamycin. Cancer Cell 2006;10:159-170. 


\section{Cellular Physiology Cell Physiol Biochem 2017;42:1755-1768 \begin{tabular}{ll|l} 
and Biochemistry & $\begin{array}{l}\text { DOI: 10.1159/000479443 } \\
\text { Published online: July 26, } 2017\end{array}$ & $\begin{array}{l}\text { @ 2017 The Author(s). Published by S. Karger AG, Basel } \\
\text { www.karger.com/cpb }\end{array}$ \\
\hline
\end{tabular}}

Bai et al.: Differential Role of RPM in Wound Healing

18 Edinger AL, Linardic CM, Chiang GG, Thompson CB, Abraham RT: Differential effects of rapamycin on mammalian target of rapamycin signaling functions in mammalian cells. Cancer Res 2003;63:8451-8460.

19 O'Reilly KE, Rojo F, She QB, Solit D, Mills GB, Smith D, Lane H, Hofmann F, Hicklin DJ, Ludwig DL, Baselga J, Rosen N: mTOR inhibition induces upstream receptor tyrosine kinase signaling and activates Akt. Cancer Res 2006;66:1500-1508.

20 Marcais A, Cherfils-Vicini J, Viant C, Degouve S, Viel S, Fenis A, Rabilloud J, Mayol K, Tavares A, Bienvenu J, Gangloff YG, Gilson E, Vivier E, Walzer T: The metabolic checkpoint kinase mTOR is essential for IL-15 signaling during the development and activation of NK cells. Nat Immunol 2014;15:749-757.

-21 Mao Y, van Hoef V, Zhang X, Wennerberg E, Lorent J, Witt K, Masvidal L, Liang S, Murray S, Larsson O, Kiessling R, Lundqvist A: IL-15 activates mTOR and primes stress-activated gene expression leading to prolonged antitumor capacity of NK cells. Blood 2016;128:1475-1489.

-22 Zhou T, Yang Z, Chen Y, Huang Z, You B, Peng Y, Chen J: Estrogen Accelerates Cutaneous Wound Healing by Promoting Proliferation of Epidermal Keratinocytes via Erk/Akt Signaling Pathway. Cell Physiol Biochem 2016;38:959-968.

23 Xu R, Luo G, Xia H, He W, Zhao J, Liu B, Tan J, Zhou J, Liu D, Wang Y, Yao Z, Zhan R, Yang S, Wu J: Novel bilayer wound dressing composed of silicone rubber with particular micropores enhanced wound reepithelialization and contraction. Biomaterials 2015;40:1-11.

24 Xu R, Xia H, He W, Li Z, Zhao J, Liu B, Wang Y, Lei Q Kong Y, Bai Y, Yao Z, Yan R, Li H, Zhan R, Yang S, Luo G, $\mathrm{Wu}$ J: Controlled water vapor transmission rate promotes wound-healing via wound re-epithelialization and contraction enhancement. Sci Rep 2016;6:24596.

-25 Calimeri T, Ferreri AJ: m-TOR inhibitors and their potential role in haematological malignancies. Br J Haematol 2017;177:684-702.

-26 Phillips BN, Wroblewski KJ: A retrospective review of oral low-dose sirolimus (rapamycin) for the treatment of active uveitis. J Ophthalmic Inflamm Infect 2011;1:29-34.

27 Wu W, He Z, Zhang Z, Yu X, Song Z, Li X: Intravitreal injection of rapamycin-loaded polymeric micelles for inhibition of ocular inflammation in rat model. Int J Pharm 2016;513:238-246.

-28 Choi YJ, Gurunathan S, Kim D, Seok JH, Park WJ, Cho SG, Park C, Song H, Seo HG, Kim JH: Rapamycin ameliorates chitosan nanoparticle-induced developmental defects of preimplantation embryos in mice. Oncotarget 2016;7:74658-74677

29 Pan T, Rawal P, Wu Y, Xie W, Jankovic J, Le W: Rapamycin protects against rotenone-induced apoptosis through autophagy induction. Neuroscience 2009;164:541-551.

-30 Blauvelt A, Asada H, Klaus-Kovtun V, Altman DJ, Lucey DR, Katz SI: Interleukin-15 mRNA is expressed by human keratinocytes Langerhans cells, and blood-derived dendritic cells and is downregulated by ultraviolet B radiation. J Invest Dermatol 1996;106:1047-1052.

31 Ali AK, Nandagopal N, Lee SH: IL-15-PI3K-AKT-mTOR: A Critical Pathway in the Life Journey of Natural Killer Cells. Front Immunol 2015;6:355.

-32 Yang G, Murashige DS, Humphrey SJ, James DE: A Positive Feedback Loop between Akt and mTORC2 via SIN1 Phosphorylation. Cell Rep 2015;12:937-943.

-33 Carr TD, DiGiovanni J, Lynch CJ, Shantz LM: Inhibition of mTOR suppresses UVB-induced keratinocyte proliferation and survival. Cancer Prev Res (Phila) 2012;5:1394-1404.

-34 Zhang HT, Wang WW, Ren LH, Zhao XX, Wang ZH, Zhuang DL, Bai YN: The mTORC2/Akt/NFкB PathwayMediated Activation of TRPC6 Participates in Adriamycin-Induced Podocyte Apoptosis. Cell Physiol Biochem 2016;40:1079-1093.

35 De Creus A, Van Beneden K, Stevenaert F, Debacker V, Plum J, Leclercq G: Developmental and functional defects of thymic and epidermal $V$ gamma 3 cells in IL-15-deficient and IFN regulatory factor-1-deficient mice. J Immunol 2002;168:6486-6493.

36 Kagimoto Y, Yamada H, Ishikawa T, Maeda N, Goshima F, Nishiyama Y, Furue M, Yoshikai Y: A regulatory role of interleukin 15 in wound healing and mucosal infection in mice. J Leukoc Biol 2008;83:165-172.

37 Macleod AS, Havran WL: Functions of skin-resident gammadelta T cells. Cell Mol Life Sci 2011;68:23992408.

38 Shen S, Alt A, Wertheimer E, Gartsbein M, Kuroki T, Ohba M, Braiman L, Sampson SR, Tennenbaum T: PKCdelta activation: a divergence point in the signaling of insulin and IGF-1-induced proliferation of skin keratinocytes. Diabetes 2001;50:255-264. 